\title{
FOLIATIONS OF CODIMENSION ONE
}

\author{
BY JOHN W. WOOD
}

\section{Communicated by Raoul Bott, February 24, 1970}

In this note we apply results of [6] to obtain some sufficient conditions for a plane field of codimension one on a manifold to be homotopic to a foliation. This and related questions on foliations are discussed in E. Thomas' survey $[5, \S 4]$ and, for open manifolds, by A. Haefliger [2]. A. V. Phillips has shown [3] that any field of codimension one on an open manifold is homotopic to a foliation.

Let $M$ be a compact riemannian manifold with boundary. It is convenient to work with the normal line field which corresponds to any plane field of codimension one. A line field is defined by a bundle monomorphism $f: \lambda \hookrightarrow \tau$ where $\lambda$ is some line bundle over $M$ and $\tau$ is the tangent bundle; we say $\lambda$ embeds in $\tau$. A homotopy of plane fields corresponds to a homotopy of bundle monomorphisms. We require $f(\lambda \mid \partial M)$ to be normal to the boundary and homotopies to be relative to the boundary. In particular, $\lambda \mid \partial M$ is trivial.

It is unknown which line bundles over $M$ embed as the normal fields of foliations. We can however prove a stable theorem. Let $p: M \times S^{1} \rightarrow M$ be the projection map.

THEOREM 1. For any line bundle $\lambda \rightarrow M, p^{*} \lambda$ embeds as the normal field of a folidation of $M \times S^{1}$.

This is in contrast to the situation in higher codimension. The normal bundle $\sigma$ of a foliation must satisfy Bott's condition that the ring generated by the rational Pontrjagin classes of $\sigma$ vanishes in dimension $>2 \operatorname{dim} \sigma$; and if $\sigma$ does not satisfy Bott's condition neither does $p^{*} \sigma$. For codimension 2 for example $p_{1}(\sigma)^{2}=0$. If $\lambda$ is the canonical line bundle over $R P^{m}$, then $p^{*} \gamma$ embeds as the normal field of a foliation of $R P^{m} \times S^{1}$ and $w_{1}\left(p^{*} \gamma\right)^{m} \neq 0$. This foliation is easily described. There is a map from the solid torus $B^{m} \times S^{1}$ onto $R P^{m} \times S^{1}$ which is a diffeomorphism on int $B^{m} \times S^{1}$ and a double cover from $S^{m-1} \times S^{1}$ to $R P^{m-1} \times S^{1} \subset R P^{m} \times S^{1}$. The Reeb foliation of $B^{m} \times S^{1}$ passes to the desired foliation of $R P^{m} \times S^{1}$.

We will need the following known fact.

Lemma 1. Let $\lambda \rightarrow M$ be a line bundle, $s$ a section transverse to the zero section, $N=s^{-1}$ (zero section), and $i: N \subset M$. Then $w_{1}(\lambda) \cap[M]=$

AMS 1969 subject classifications. Primary 5736; Secondary 5730.

Key words and phrases. Foliation, line field, homotopy of line fields. 
$i_{*}[N]$ and $\lambda \mid N=\nu$ (as bundles) where $\nu$ is the normal bundle of $N$ in $M$. If $\lambda \mid \partial M$ is trivial we may take $N C$ int $M$.

Proof of Theorem 1. Let $s$ and $N$ be as in the lemma with $N \subset$ int $M$ and let $\hat{M}$ be $M$ cut along $N$. The construction of [ $6 \mathrm{p}$. 339] gives a foliation of $\hat{M} \times S^{1}$ with a vector field normal to the foliation and inward normal on $\partial \hat{M} \times S^{1}$. Gluing along $N \times S^{1}$ we obtain a foliation of $M \times S^{1}$ and a section $t$ of the normal line field transverse to the zero section and such that $t^{-1}$ (zero section) $=N \times S^{1}$. Note that $s$ gives a section of $p^{*} \lambda$ with the same properties. By the lemma (applied to $\left.M \times S^{1}\right) p^{*} \lambda$ is equivalent to the normal line field of the foliation (since the line bundles are classified by $w_{1}$ and hence by $\left.\left[N \times S^{1}\right]\right)$.

The following two results for the case where the normal line field is trivial are essentially contained in [6].

THEOREM 2. If $f: \epsilon^{1 \subset} \hookrightarrow \tau\left(M \times S^{1}\right)$, then $f$ is homotopic to the normal field of a foliation.

THEOREM 3. If $\partial M^{3}$ is a union of tori (or empty), then $f: \epsilon^{1 \subset} \hookrightarrow \tau M^{3}$ is homotopic to the normal field of a foliation.

Let $v$ be a nonvanishing vector field in $f\left(\epsilon^{1}\right)$. If $v$ is inward normal on all boundary components then these results are just Corollary 9.4 and Proposition 10.3 of [6]. Otherwise suppose $v$ is outward normal along $N \subset \partial M^{3}$. After a homotopy we may assume $v=-\partial / \partial t$ on a collar $N \times[0,2)$ of $N$ in $M$. Since $N$ has a nonvanishing vector field, $v \mid N \times\{1\}$ is homotopic to $+\partial / \partial t \mid N \times\{1\}$. Thus after a homotopy $-v$ is inward normal on $N \times[0,1], v$ is inward normal on $M-N$ $\times[0,1)$, and we are reduced to the previous case. The case of $M \times S^{1}$ differs only in notation.

If $\lambda \rightarrow M$ is any line bundle, there is an embedding $f: \lambda \hookrightarrow \tau M \oplus \epsilon^{1}$. Identifying $\tau\left(M \times S^{1}\right)=p^{*}\left(\tau M \oplus \epsilon^{1}\right), f$ corresponds to an embedding $p^{*} f: p^{*} \lambda \hookrightarrow \tau\left(M \times S^{1}\right)$. This is the type of embedding that occurs in Theorem 1 .

TheOREM 4. For any $f: \lambda \hookrightarrow \tau M \oplus \epsilon^{1}, p^{*} f$ is homotopic to the normal field of a foliation.

LeMma 2. If $\lambda^{1}$ and $\mu^{n}$ are bundles over $X$, there is a one-one correspondence between bundle maps $\lambda \hookrightarrow \mu$ and bundle maps $\epsilon^{1} \hookrightarrow \lambda \otimes \mu$ (i.e. nonvanishing sections of $\lambda \otimes \mu$ ) and also between homotopy classes.

This is well known and follows by tensoring with $\lambda$ from the fact that $\lambda \otimes \lambda=\epsilon^{1}$.

Proof of Theorem 4. Let $N$ and $\nu$ be as in Lemma 1 ; we regard $\nu$ 
as a line bundle on $N$ with a fixed embedding $\nu \subseteq \tau M \mid N$. As subbundles of $\left(\tau M \oplus \epsilon^{1}\right)|N \supset \tau M| N, \lambda \mid N$ and $\nu$ are homotopic by Lemma 2. Hence $p^{*}(\lambda \mid N)$ is homotopic to $p^{*} \nu$ in $p^{*}\left(\left(\tau M \oplus \epsilon^{1}\right) \mid N\right)$. Under the identification of $p^{*}\left(\tau M \oplus \epsilon^{1}\right)$ with $\tau\left(M \times S^{1}\right), p^{*} \nu$ is identified with the normal bundle of $N \times S^{1}$ in $M \times S^{1}$. Hence $p^{*} \lambda$ is homotopic to $\mu$ on $M \times S^{1}$ with $\mu \mid N \times S^{1}$ normal to $N \times S^{1}$. Let $\hat{M}$ be $M$ cut along $N$; then $\mu$ is trivial on $\hat{M} \times S^{1}$ so Theorem 2 implies the result.

In particular, if $f: \lambda \subset \tau M$ then, after crossing with $S^{1}, p^{*} f$ is homotopic to the normal field of a foliation. To study more general embeddings of $p^{*} \lambda$ in $\tau\left(M \times S^{1}\right)$ we need the following result of obstruction theory.

Lemma 3. Let $M^{m}$ be orientable, $m$ odd, $i: N C$ int $M, \chi(M-N)=0$, $i_{*}[N] \neq 0$, and $\lambda \rightarrow M$ a line bundle with $w_{1}(\lambda) \cap[M]=i_{*}[N]$. Then $\lambda$ embeds in $\tau M$ and for any embedding $\lambda \mid N$ is homotopic to the normal bundle of $N$ in $M$.

Proof. Let $\hat{M}$ be $M$ cut along $N \cdot \chi(\hat{M})=0$ so $\hat{M}$ has a nonvanishing vector field normal along the boundary. This vector field provides a line field on $M$ which is an embedding of $\lambda$ (see the proof of Theorem 1). There is a corresponding section $f: \epsilon^{1 \hookrightarrow} \hookrightarrow \lambda \otimes \tau$. We claim that any section $g: \epsilon^{1}|N \hookrightarrow(\lambda \otimes \tau)| N$ which extends over $M$ is homotopic to $f \mid N$. Homotopy classes of sections of $(\lambda \otimes \tau) \mid N$ are classified by elements $d(f \mid N, g) \in H^{m-1}(N ; \mathbb{C} \mid N)$ where $\mathbb{e}$ is the bundle of integer coefficients on $M$ twisted by $w_{1}(\lambda \otimes \tau): \pi_{1}(M) \rightarrow$ Aut $Z,[4, \S 37]$. Since $\left.w_{1}(\lambda \otimes \tau)=m w_{1}(\lambda)+w_{1}(\tau), \quad w_{1}(\lambda \otimes \tau) \mid N\right)=w_{1}(N)$ and $\mathcal{e} \mid N$ is the orientation bundle of $N$. Hence $H^{m-1}(N ; \mathrm{e} \mid N)=\boldsymbol{Z}$. Those classes which extend over $M$ are in the image of $i^{*}$ in the sequence

$$
H^{m-1}(M ; \mathfrak{e}) \stackrel{i^{*}}{\rightarrow} H^{m-1}(N ; \mathfrak{e} \mid N) \rightarrow H^{m}(M, N ; \mathfrak{e}) \rightarrow H^{m}(M ; \mathfrak{e}) \rightarrow 0 .
$$

$H^{m}(M, N ; \mathfrak{C})=\boldsymbol{Z}$ using a tubular neighborhood, excision, and the fact that $\lambda \mid M-N$ is trivial.

$$
\begin{array}{rll}
H^{m}(M ; \mathcal{C})=Z & \text { if } w_{1}(\lambda \otimes \tau)=0 & \text { iff } w_{1}(\lambda)=0 \\
=Z_{2} & \text { if } w_{1}(\lambda \otimes \tau) \neq 0 & \text { iff } w_{1}(\lambda) \neq 0 .
\end{array}
$$

Since $i_{*}[N] \neq 0$, we see that $i^{*}=0$. Thus if $g$ extends, then $g \simeq f \mid N$, which completes the proof.

THEOREM 5. If $M^{m}$ is orientable, $m$ even, and $\lambda \rightarrow M$ is a line bundle, then any $f: p^{*} \lambda \subset \tau\left(M \times S^{1}\right)$ is homotopic to a line field normal to a foliation.

Proof. We may assume $\lambda$ is nontrivial. Let $N$ be as in Lemma 1 and 
apply Lemma 3 to $N \times S^{1} \subset M \times S^{1}$ to get $f \simeq g$ with $g\left(p^{*}(\lambda \mid N)\right)$ normal to $N \times S^{1}$ in $M \times S^{1}$. Then cut and use Theorem 2 .

THEOREM 6. Let $M^{3}$ be an orientable 3-manifold with $\partial M^{3}$ a union of tori (or empty). Let $i: N^{2} \subset$ int $M^{3}$ ( $N^{2}$ not necessarily connected or orieniable), and $\chi(N)=0$. If $w_{1}(\lambda) \cap[M]=i_{*}[N]$, then any $f: \lambda \hookrightarrow \tau M$ is homotopic to a field normal to a foliation.

Proof. Apply Lemma 3 and Theorem 3 as above.

Any line bundle $\lambda$ on a closed 3 -manifold $M$ is classified by $w_{1}(\lambda)$ or by its Poincaré dual $u \in H_{2}\left(M^{3} ; \boldsymbol{Z}_{2}\right)$, and $u$ is represented by some surface $N$. If $N$ is connected and nonorientable, then $N=R P^{2}$ $\# \cdots \# R P^{2}$ ( $h$ times) and $\lambda$ embeds in $\tau M$ if and only if $w_{1}(\lambda)^{3} \neq 0$ $[6,11.4]$ if and only if $h$ is even [1, p. 88]. In general $\lambda \subset \tau M$ if and only if the sum of the genera of the nonorientable components of $N$ is even. If $u$ is represented by an even number of disjoint embedded $R P^{2}$ 's, then by surgery along arcs connecting them in pairs, $u$ can be represented by disjoint Klein bottles. If $u$ is represented by an embedded $S^{2}$, then by adding a small handle $u$ can be represented by $S^{1} \times S^{1}$. This gives the following.

CoROLlary 1. If any element of $H_{2}\left(M^{3} ; Z_{2}\right)$ can be represented by a disjoint set of embedded surfaces with Euler characteristics $\geqq 0$, then for any $\lambda \rightarrow M$, any $f: \lambda \subseteq \tau$ is homotopic to the normal line field of a foliation.

Notice that if $M_{1}$ and $M_{2}$ satisfy the hypothesis of Corollary 1 then so does $M_{1} \# M_{2}$.

CoRollary 2. The conclusion of Corollary 1 holds for any connected sum of the following manifolds: $S^{1} \times S^{2}, R P^{3}, S^{1} \times S^{1} \times S^{1}$, and the lens spaces $L(4 k, 2 k-1)$.

See [1] for the fact that the Klein bottle embeds in $L(4 k, 2 k-1)$ and also for the statements below.

If $M^{2}$ is orientable of genus $g$, then $M^{2} \times S^{1}$ has a line field corresponding to the class represented by $M^{2} \times\{*\}$ and this class cannot be represented by an orientable surface of genus $<g$ or a nonorientable surface of genus $<2 g+2$. The other line bundles on $M^{2} \times S^{1}$ can be embedded normal to a foliation by Theorem 5 or 6 .

Question 1. Can all line bundles on $M^{2} \times S^{1}, M^{2}$ orientable of genus $\geqq 2$, be embedded as the normal fields of foliations?

For the lens space $L(p, q)$, if $p$ is odd there are no nontrivial line bundles. If $p=4 k+2$ there are no nontrivial line fields. If $p=4 k$ there are nontrivial line fields, but $L(4 k, 2 k-1)$ is the only space in 
which the Klein bottle embeds. (The torus always bounds.) Thus we also leave unanswered

Question 2. Does the nontrivial line bundle on $L(8,1)$ occur as the normal field of a foliation?

\section{REFERENCES}

1. G. E. Bredon and J. W. Wood, Non-orientable surfaces in orientable 3-manifolds. Invent. Math. 7 (1969), 83-110.

2. A. Haefliger, Feuilletages sur les variêtés ouvertes, Topology 9 (1970), 183-194.

3. A. V. Phillips, Foliations on open manifolds. I, Comment. Math. Helv. 43 (1968), 204-211. MR 37 \#4829.

4. N. Steenrod, The topology of fibre bundles, Princeton Math. Series, vol. 14, Princeton Univ. Press, Princeton, N. J., 1951. MR 12, 522.

5. E. Thomas, Vector fields on manifolds, Bull. Amer. Math. Soc. 75 (1969), 643683. MR $39 \# 3522$.

6. J. W. Wood, Foliations on 3-manifolds, Ann. of Math. (2) 89 (1969), 336-358,

Institute for Advanced Study, Princeton, New Jersey 08540 